\title{
Developing the Next Generation Shell Buckling Design Factors and Technologies
}

\author{
Mark W. Hilburger* \\ NASA Langley Research Center, Hampton, Virginia, 23681, USA
}

\begin{abstract}
NASA's Shell Buckling Knockdown Factor (SBKF) Project was established in the spring of 2007 by the NASA Engineering and Safety Center (NESC) in collaboration with the Constellation Program and Exploration Systems Mission Directorate. The SBKF project has the current goal of developing less-conservative, robust shell buckling design factors (a.k.a. knockdown factors) and design and analysis technologies for light-weight stiffened metallic launch vehicle (LV) structures. Preliminary design studies indicate that implementation of these new knockdown factors can enable significant reductions in mass and mass-growth in these vehicles and can help mitigate some of NASA's LV development and performance risks. In particular, it is expected that the results from this project will help reduce the reliance on testing, provide high-fidelity estimates of structural performance, reliability, robustness, and enable increased payload capability. The SBKF project objectives and approach used to develop and validate new design technologies are presented, and provide a glimpse into the future of design of the next generation of buckling-critical launch vehicle structures.
\end{abstract}

\section{Introduction}

$\mathrm{H}$ igh-performance aerospace shell structures are inherently thin walled because of weight and performance considerations and are often subjected to destabilizing loads. Thus, buckling is an important and often critical consideration in the design of these structures and reliable, validated design criteria for thin-walled shells are needed, especially for shells fabricated with advanced materials and manufacturing techniques. Unfortunately, the current design guidelines have not been updated since they were first published in the late 1960s and may not be able to take full advantage of modern materials, precision manufacturing processes, and new structural concepts.

From the 1920s to the early 1970s, many shell buckling experiments were conducted in an effort to understand the complex buckling behavior exhibited by thin-walled cylindrical shells and to provide data to correlate with new theories of elastic stability of these shells. Typically, the experiments yielded buckling loads that were substantially lower than the corresponding analytical predictions, which were based on simplified linear bifurcation analyses of geometrically perfect shells with nominal dimensions and idealized boundary conditions. The seminal works by von Kármán and Tsien ${ }^{1}$, by Donnell and $\mathrm{Wan}^{2}$, and by Koiter ${ }^{3}$ identified small deviations from the idealized geometry of a shell, known as initial geometric imperfections, as a primary source of the discrepancy between corresponding analytical predictions and

\footnotetext{
* Senior Research Engineer, Structural Mechanics and Concepts Branch, Hampton, VA, 23681, USA, Senior Member AIAA.
} 
experimental results. However, the computational tools and capabilities at that time could not perform the nonlinear analyses needed to assess the effects of these imperfections on the buckling behavior of thin-walled shells. Thus, buckling design allowables were determined by establishing lower bounds to test data. Specifically, empirical design factors, that have become known as knockdown factors, were determined and were to be used in conjunction with linear bifurcation analyses for simply supported shells; that is, these empirical factors were used to "knock down" the value of the un-conservative simplified analytical prediction. This approach to shell buckling design has proved satisfactory for most design purposes and remains prominent in industry practice, as evidenced by the extensive use of the NASA space vehicle design criteria and recomendations. ${ }^{4-8}$

However, there are many limitations to the current design recommendations for buckling-critical cylinders that have led to a recent reinvestment in shell buckling research and design technology development by several groups. ${ }^{9-12}$ The primary limitations in the current design practice, cited by these R\&D groups, includes the following, in no particular order:

- Knockdown factors used in the design of aerospace-quality shell structures are typically overly conservative as indicated by recent test results

- Pedigree of the test data used to develop the design knockdown factors is often difficult to assess, and in some cases undocumented and thus critical reviews of the existing data are limited

- Design information is not available for shells constructed from modern materials and manufacturing processes such as composite shells or friction-stir-welded aluminum alloy shells

- The effects of boundary conditions and shell length are assumed to be negligible

- The design recommendations and data do not provide information necessary to quantify robustness and reliability

Many of these new research and development activities are attempting to address some of these limitations and show significant promise and technical merit. If successful, the results of these activities may likely form the basis for the next generation of shell buckling design factors and recommendations.

This paper will provide an overview of just such an activity at NASA that is assessing and updating the state of the art in shell buckling design and analysis, the Shell Buckling Knockdown Factor (SBKF) Project. The SBKF Project was established in 2007 by the NASA Engineering and Safety Center (NESC) in collaboration with NASA's Constellation Program and Exploration Systems Mission Directorate. The SBKF Project has the goal of developing improved (i.e., lessconservative, robust), shell buckling design factors (a.k.a. knockdown factors) and design and analysis technologies for launch vehicle (LV) structures. Preliminary design studies indicate that the implementation of these new knockdown factors can enable significant reductions in mass and mass-growth in these vehicles and can help mitigate some of NASA's LV development and performance risks.

The paper is intended to provide the reader with an overview of the state of the art in shell buckling design and analysis and a high-level understanding of the SBKF Project objectives, approach, and expected outcome. To this end, the paper will first provide a historical review of 
the current design recommendations for buckling-critical stiffened cylindrical shell structures, identify some of their limitations relative to the design of modern launch vehicle structures, and identify areas for improvement. Then, the SBKF Project objective and technical approach and expected benefits are discussed.

\section{Current Design Practice}

The theoretical buckling load of a given stiffened cylinder design is predicted by performing a linear bifurcation buckling analysis of a geometrically perfect, uniform structure with smeared stiffener properties, simply supported boundary conditions and is subjected to a uniform axial compression load. This theoretical buckling load is then reduced, or knocked down, by applying an empirical knockdown factor to account for the differences between theory and test. Linear bifurcation buckling analyses can be performed using finite element (FE) methods or by using closed-form solutions such as those found in Section 4.3 of NASA SP$8007^{4}$ for ring and stringer (orthogrid) stiffened cylinders and in Section 4.2 of the Isogrid Design Handbook (IDH) NASA CR- $124075^{8}$ for isogrid-stiffened cylinders.

For cylinders with "closely spaced, moderately large stiffeners", NASA SP-8007 recommends that a knockdown factor (KDF) of $\mathbf{0 . 7 5}$ be applied to the predicted buckling load of cylinders subjected to uniform compression and bending loads. This KDF is a lower bound to the test data reported in Refs 13-20 and is shown in Figure 1 as a red line. Similarly, NASA TN D-5561 (the basis for the knockdown factor used in Ref 8) recommends that a knockdown factor of $\mathbf{0 . 6 5}$ be applied to the predicted buckling load of a stiffened cylinder subjected to uniform compression or bending loads. This KDF is a lower bound to the test data reported in Refs 13, and 21-25 and is shown in Figure 1 as a green line. It should be noted that these design approaches for stiffened cylinders given in SP-8007 and TN D-5561 are similar, however, the recommended KDF values are different because they are based on different test data sets. (It is not clear why this came about since both publications have a common author and were written around the same time period...)

It should also be noted that the commonly used knockdown factor equations (Eqs. 45 and 47) in NASA SP-8007 are lower bounds to test data for unstiffened orthotropic cylinders subjected to axial compression and bending loads, respectively. The equation for a compression-loaded cylinder KDF (Eq. 45) is

$$
\gamma=1-0.901\left(1-e^{-\varphi}\right)
$$

and the equation for cylinders in bending (Eq. 47) is

$$
\gamma=1-0.731\left(1-e^{-\varphi}\right)
$$

where $\gamma$ is the KDF and

$$
\varphi=\frac{1}{29.8}\left[\frac{R}{\sqrt[4]{\frac{D_{11} D_{22}}{A_{11} A_{22}}}}\right]
$$

American Institute of Aeronautics and Astronautics 
where $R$ is the radius of the cylinder and $A_{11}$ and $A_{22}$ are longitudinal and circumferential membrane stiffnesses, and $D_{11}$ and $D_{22}$ are longitudinal and circumferential bending stiffnesses of the stiffened skin, respectively. These knockdown factor equations are intended to be used in the design of orthotropic unstiffened cylinders only, but are often used for the design of stiffened shells presumably because they typically provide the most conservative KDF values. For example, Eq. 45 would give KDF values that range from $\mathbf{0 . 6 8}$ to $\mathbf{0 . 5 2}$ for typical orthogrid and isogrid cylinder designs with $\mathbf{5 0}<\boldsymbol{R} / \boldsymbol{t}_{\text {eff }}<\mathbf{1 5 0}$ subjected to compression loads. Similarly, Eq. 47 would give KDF values that range from $\mathbf{0 . 7 4}$ to $\mathbf{0 . 6 1}$ for the same orthogrid and isogrid stiffened cylinder designs subjected to bending loads. The SP-8007 KDF recommendation for compression-loaded unstiffened shells is indicated by the blue curve in Figure 1.

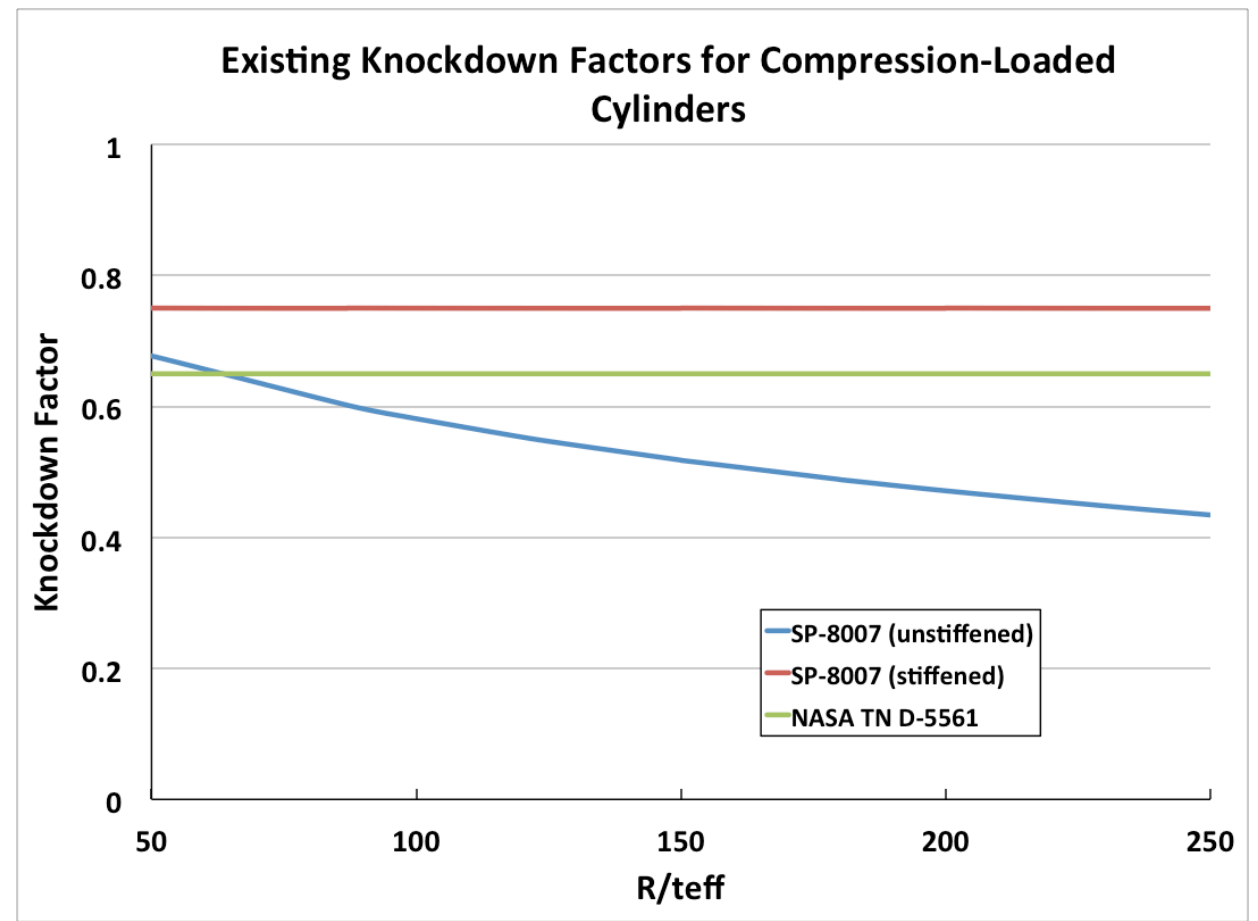

Figure 1. Current recommended buckling knockdown factor values from NASA SP-8007 and NASA TN D5561.

The test programs reported in Refs. 13-24, identified previously, were originally conducted in order to generate data for correlation with new theories of elastic stability for stiffened cylinders. In particular, the effects of stiffener configuration (e.g., ring, stringer, orthogrid, isogrid, etc.), stiffener eccentricity (i.e., internal versus external stiffeners), and failure mode coupling (local buckling and stiffener crippling) on the buckling behavior and imperfection sensitivity of stiffened cylinders were not well understood at the time. To this end, a variety of stiffened cylinder designs were studied. Four of the references $(13,14,22$, and 23$)$ are on ringstiffened corrugated cylinders similar to those used on the Apollo-era launch vehicles, three references $(15,16$, and 18) are on stringer-stiffened cylinders, two references $(17$ and 20) are on ring-stiffened cylinders, two references (19 and 24) on ring and stringer stiffened cylinders and one reference on $45^{\circ}$ waffle-stiffened cylinders (Ref. 21). Seven of the eleven cylinder configurations are built-up structures in which the stiffeners are attached to the skin using 
discrete fasteners such as rivets or bolts. The other cylinders have integrally machined blade stiffeners. The loading conditions considered in these test reports include various combinations of axial compression, bending and internal pressure. A summary of the twelve references, the structural configurations, loading conditions, and corresponding ranges of knockdown factors is provided in Table 1. KDF data from Ref 14 were not available when this paper was written. An in-depth review of the test data in these reports was recently performed by the SBKF Project but is beyond the scope of this paper. However, some background information and critical findings are presented in the remainder of this section to support subsequent recommendations on the applicability of this data in the design of modern launch vehicle structural concepts.

\begin{tabular}{|c|c|c|c|}
\hline Ref. \# & Construction & Loading & Range of KDF \\
\hline 13 & Ring-stiffened corrugated & Comp & $0.88-1.1$ \\
\hline 14 & Ring-stiffened corrugated & Comp, Bending & NA \\
\hline 15 & Longitudinally stiffened w/ Z stringers & Comp & $0.91-0.98$ \\
\hline 16 & Longitudinally stiffened $\mathrm{w} / \mathrm{Z}$ stringers & Comp & $0.86-1.08$ \\
\hline 17 & Integral ring stiffened & Comp & $0.80-0.96$ \\
\hline 18 & Longitudinally stiffened w/ Z stringers & Comp & $0.70-0.95$ \\
\hline 19 & Integral ring/stringer/grid stiffened & Comp & $0.74-1.16$ \\
\hline 20 & Integral rings/stinger stiffened & Comp & $0.7-1.2$ \\
\hline 21 & Integral $45^{\circ}$ waffle-grid stiffened & $\begin{array}{l}\text { Comp, Bend, Int. } \\
\text { Press }\end{array}$ & $0.68-1.08$ \\
\hline 22 & Ring-stiffened corrugated & Bending & $0.88-1.02$ \\
\hline 23 & Ring-stiffened corrugated & Bending & $0.70-0.72$ \\
\hline 24 & Ring \& stringer stiffened & Bending & $0.86-1.06$ \\
\hline
\end{tabular}

Table 1. Summary of references used to define shell buckling knockdown factors for stiffened cylinders in NASA SP-8007 and NASA TN D-5561.

The majority of these test programs ( 7 of 12) were conducted by or overseen by NASA engineers and appear to be of relatively high quality. The quality of the other test reports is somewhat mixed but provides some useful data and information. In general, most of the researchers, having benefited from previous testing conducted on unstiffened imperfectionsensitive shells, acknowledge the importance of designing and fabricating high-quality test articles and conducting careful tests. Many of the reports provide information that is useful in assessing the overall quality of the test data including manufacturing tolerances and measurements, test article design approach, and test set-up and data. In addition, some of the test results are accompanied by in-depth discussions on the physics of the behaviors observed and their correlation with theoretical predictions. Overall, it was found that many of the results presented in these references are useful in identifying general trends associated with the buckling response and imperfection sensitivity of the various stiffened cylinders considered (all of these behavioral trends are now relatively well understood). In particular:

1. stiffened cylinders are typically much less imperfection sensitive than unstiffened cylinders. Stiffened cylinders show a reduced amount of scatter in the test data and the buckling loads are typically much closer to the predicted loads and thus require a less severe buckling knockdown factor than unstiffened shells.

2. imperfection sensitivity decreases as the as the overall percentage of stiffening mass increases (e.g., Ref 20) 
3. structural efficiency and imperfection sensitivity can vary significantly depending on the stiffening concept, e.g., internal vs. external stiffeners, ring vs. stringer, etc. (e.g., Ref 19)

4. imperfection sensitivity can vary significantly depending on the combination of orthotropy and boundary conditions (i.e., strong coupling between orthotropy and boundary conditions)

Most of the test data from the built-up stiffened cylinders also follow some of these same trends. However, additional behavioral characteristics that can affect global buckling and imperfection sensitivity are briefly described next.

Test data reported in references 16 and 18 correspond to shells that were designed to exhibit skin buckling prior to global buckling. Special theories that can account for the effects of buckled skins on the effective stiffnesses and global buckling were successfully implemented and produced good correlation with the test results. However, the effects of local skin buckling on the imperfection sensitivity have not been quantified for the cylinders in question. It is likely that the local skin buckling reduces the imperfection sensitivity of the shell because the buckling pattern itself becomes the dominant geometric feature as compared to a smaller magnitude initial geometric imperfections, and thus a KDF that is derived from these data could be unconservative.

Test data for ring-stiffened corrugated shells reported in references 13, 14, 22, and 23 indicate that nonlinear pre-buckling bending deformations form in the corrugated skin and appear to have an effect on the global buckling response of the shell. Several reports made note of the behavior, but, the reason for the pre-buckling bending deformations was not identified and the effects of these deformations on the buckling loads and imperfection sensitivity were not quantified. If this behavior is similar to a local skin buckling response then it may influence the imperfection sensitivity and global buckling load. The complex built-up nature of this type of construction (flexibility of the ring attachments and complex load paths between the corrugated skin and the rings) will make any assessment of the observed behavior difficult without resorting to high-fidelity geometrically nonlinear structural simulations.

In contrast, the reports on the $45^{\circ}$ waffle-stiffened cylinders in Ref 21 and the ringstiffened corrugated cylinders in Ref 23 are not particularly useful in establishing response trends for stiffened cylinders. In fact, the technical content of these two reports is deficient to the extent that it is difficult to assess the relative quality of the data one way or the other. Of particular concern is the fact that both data sets appear to contain anomalous results that go unexplained in the test reports. For example, some of the test results in Ref 21 indicate that local skin buckling preceded global buckling in some of the tests even though a $40 \%$ margin of safety on local skin buckling was calculated for these designs. In addition, the global buckling mode predicted from a linear buckling analysis was shown to have a similar wave length to that of the stiffener spacing. This can cause several undesirable outcomes including over estimation of theoretical buckling load (associated with a potential violation of smeared stiffener theory which would over estimate the structural stiffness) and the possibility of triggering unexpected local-global buckling interactions in the test article (where the wave length of the global mode could excite or couple with a local buckling mode in the pre-buckling range of loading). A private communication with a former Douglas Aircraft Engineer indicated that there was an internal debate as to whether this questionable test data should be included in the report because of concerns over local skin buckling. Unfortunately, Ref 21 does not provide any information that 
could be used to conduct a critical assessment of the data in question. Similarly, two buckling tests on ring-stiffened corrugated cylinders are presented in Ref 23. These test were intended to be very similar to other buckling test conducted at $\mathrm{NASA}^{22}$ and used the same structural configurations and test facility. However, the buckling loads reported in Ref 23 are approximately $20 \%$ lower than the other buckling loads reported in Ref 21 . Unfortunately, the report does not provide any information that can be used to identify the possible reasons for the discrepancies. However, the fact that the buckling loads reported in Ref 23 fall within several percent of each other but are approximately $20 \%$ lower than the results from the earlier tests ${ }^{21}$ suggests a systematic problem with these tests.

Given the review of the current knockdown factors and the associated test data, the following conclusions are drawn:

1. The test results presented in references 13-24 are useful in determining general trends and bounds associated with the buckling response and imperfection sensitivity of the various types of stiffened cylinders considered. However, the test articles are not representative of modern aerospace-quality stiffened cylinders and thus might not be directly applicable in design due to manufacturing tolerances, boundary conditions and structural scale. Additional work is necessary to draw more detailed conclusions on their direct applicability.

2. Data from stiffened cylinders that exhibit local skin buckling prior to global buckling should be treated separately from shells that exhibit global buckling only, due to the possibility of providing un-conservative estimates of global buckling loads and imperfection sensitivity.

3. Ring-stiffened corrugated cylinders are complex built-up structures that appear to exhibit local prebuckling bending deformations in the corrugated skin. These shells should be treated separately since it is unknown how these bending deformations affect the buckling and imperfection sensitivity of these shells.

4. Test data from refs 21 and 23 should be excluded from the design data base due to a lack of confidence in the quality of the test results

5. Future knockdown factors should account for and include parametric representation of all critical shell and stiffener stiffnesses and eccentricities, length effects, and boundary conditions

6. Clear technical rationale should be provided when test data and KDFs derived from a specific stiffened cylinder configuration are used on different stiffened cylinders. In particular, rationale should include quantitative results that indicate similarity in buckling behavior and imperfection sensitivity

\section{Overview of SBKF Objectives and Approach}

An overview of NASA's SBKF Project is presented in this section. First, the current program objectives are presented. Then the general approach used to meet the project objectives is described. Finally, a brief description of the theory and rationale for the new analysis-based knockdown factors is presented.

\section{Objective}

The current objective of the SBKF project is to develop and validate new analysis-based knockdown factors and design recommendations for buckling-critical integrally-stiffened 
cylindrical shells. These new factors are being developed first for a NASA-specific launch vehicle design space and will then be extended to a larger design space at a later date. These new factors and design recommendations will address and overcome many of the limitations that exist currently as identified in the previous sections. In particular, these factors and recommendations will be tailored for specific modern light-weight integrally-stiffened orthogrid and isogrid metallic designs. The factors will be explicit functions of shell geometry, orthotropy (i.e., stiffener pattern and eccentricity), manufacturing tolerances and boundary conditions. In addition, factors will account for the effects of relevant combined mechanical, pressure, and thermal loads common to launch vehicles and manufacturing specific structural details such as longitudinal joints. It is expected that these new factors will reduce structural mass and massgrowth potential in the design of these structures, and provide designers and decision-makers improved design information earlier in the design cycle that can help reduce the chance of getting locked into a poor design. The SBKF project will also provide validated analysis tools and guidelines that can reduce the risk of producing erroneous buckling predictions and help reduce the reliance on structural testing by enabling high-fidelity buckling predictions of as-built hardware. More details on the implementation of the new factors and their expected benefits will be provided later in the paper.

\section{Approach}

The SBKF Project employs a four-part approach to achieve its technology development and implementation goals and is briefly described here.

First, SBKF conducts extensive design trades studies to assess sensitivities to design factors, materials, and structural concepts as well as detailed analyses to determine performance and weight drivers associated with structural details such as welds and joints, cutouts, and other discontinuities and details that may affect buckling. These trade studies are performed at the component and vehicle level to help assess performance trends and component interaction effects, respectively. Data from these trade studies help target high-payoff structural configurations as well as to provide a baseline for assessing the technologies as they are developed. $^{25-27}$

Second, SBKF is engaged in numerical and experimental studies to assess the effects of other failure modes that are common in the design of buckling-critical shell structures such as local skin buckling, stiffener crippling as well as the effects of elastic boundary conditions, and combined mechanical, thermal, and pressure loads. The primary reason for these studies is to reduce the implementation risk of the new knockdown factors. More specifically, it was determined, during the initial planning phase of the Project, that one potential risk in implementing less-conservative knockdown factors would be to reduce the robustness of a design to other effects that are currently "accounted for" by the overly-conservative design factors. $^{28-29}$

Third, SBKF is developing and validating new analysis-based knockdown factors, and design and analysis tools and recommendations for selected structural concepts (the final product of the SBKF project). Results from the preliminary trade studies and detailed analyses have been used to guide the development of new knockdown factors and design technologies. Coordination with NASA's launch vehicle development programs and interactions with industry partners have 
helped define the design space of interest and determine appropriate design and implementation assumptions. Currently, SBKF is focusing on the development of knockdown factor equations for integrally-stiffened, friction-stir welded, metallic cylindrical structures. These new factors are being validated with sub-scale and full-scale buckling tests of representative integrally-stiffened cylinders. These new knockdown factors will be based on the results from high-fidelity analysis tools that have been rigorously validated through sub-scale and full-scale structural tests. Some details on the analysis models and the technical rationale that supports the development of these new knockdown factors are presented in the next section.

Finally, SBKF understands the importance of having a technology implementation strategy that is closely coupled with NASA's launch vehicle development programs. To this end, SBKF has interacted directly with NASA stakeholders, e.g., designers, managers, discipline experts, and chief engineers, through the use of Workshops ${ }^{30}$ and Peer Reviews to help plan and review the SBKF project plan and help to ensure successful technology development and implementation. These interactions have helped build a strong working relationship between research and design teams and have provided significant feedback to maximize the potential benefits and success of the SBKF project. What adds to the challenge of the technology implementation process, and is quite possibly the biggest challenge for the project, is the fact that the implementation of new SBKF technologies will require some change in the design paradigm that has been in place since the 1960s. To this end, much of the interactions between the technology developers within SBKF and stakeholders go beyond the technical debates and discussions and are often focused on forming a necessary level of understanding and trust in the new factors, recommendations and rationale.

\section{Analysis-Based Knockdown Factors}

Analysis-based knockdown factors are now becoming a viable replacement for the test-based knockdown factors used currently. More specifically, improved nonlinear structural analysis tools and improved theories of elastic stability and imperfection sensitivity in shell structures are enabling high-fidelity predictions of the buckling response of thin-walled compression-loaded cylindrical shells ${ }^{31}$. These high-fidelity predictions and analysis tools are the foundation for the new analysis-based knockdown factors being developed by SBKF. In addition, advanced testing technologies are being used to obtain critical data required to rigorously validate these predictions and corresponding analysis-based factors. ${ }^{32}$ One of the key attributes of the new factors and their method of development is that specific design features can be isolated and their effects on buckling can be characterized. In turn, the resulting design factor will have the fidelity to account for selected design features that are relevant for the design in question. For example, SBKF is currently developing and validating lower-bound knockdown factor equations that will account for six design features, which are as follows:

1. Geometric imperfection (shape and amplitude)

2. Loading non-uniformity associated with manufacturing and fit-up tolerances (shape and amplitude)

3. Stiffener pattern variation (i.e., orthotropy)

4. Combined mechanical, thermal, and pressure loads

5. Cylinder geometry (radius, and length)

6. Longitudinal welded joints in metallic structures (a.k.a., weld lands) 
In this way, the KDF can be tailored to the specific design and can evolve as the design matures and more information becomes available.

The geometric imperfection used for the analysis-based KDF can take several different forms. A traditional analysis-based approach would typically use a set of one or more eigen-mode shapes to generate an imperfection pattern and then a range of imperfection amplitudes would be assumed to generate a lower bound buckling load (e.g., see filled triangle in Figure 2a). This approach has some advantages in that it is simple to implement and typically gives a conservative lower-bound estimate of the buckling load. However, the eigen-mode imperfection shape is not a pattern typically observed in as-built structures and the choice of mode shapes to include in the simulated imperfection is somewhat arbitrary. In addition, the eigen-mode imperfection typically causes a significant reduction in the pre-buckling stiffness of the shell as shown in Figure 2b, which is not seen in actual tests.

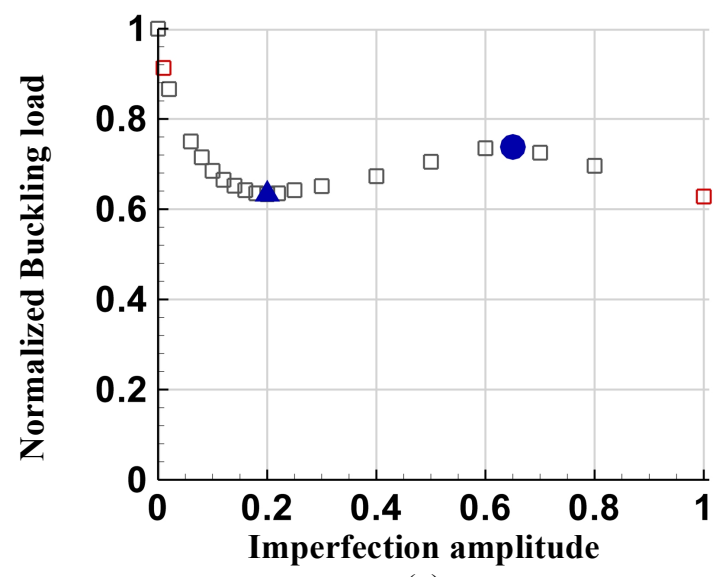

(a)

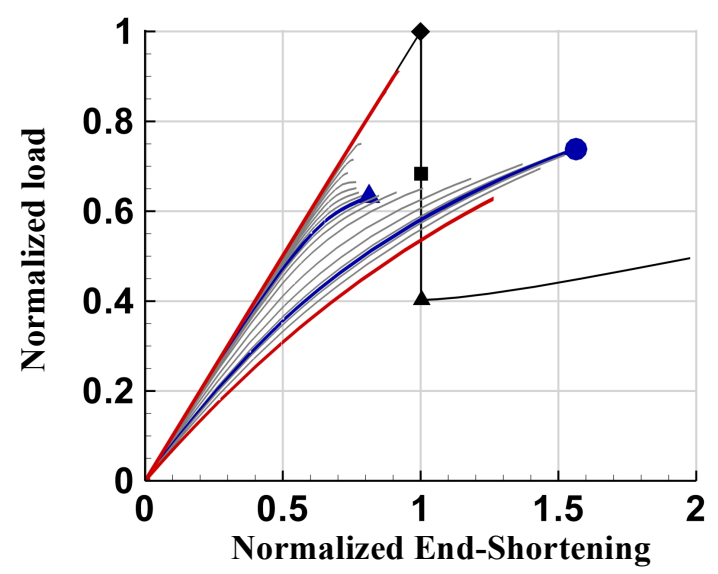

(b)

Figure 2. Predicted buckling loads (a), and load-end-shortening curves (b) for a compression-loaded cylinder for various imperfection amplitudes.

In contrast, an alternate approach is being pursued in the SBKF project in which a lateral perturbation load is used to create a local dimple-shaped imperfection in the shell wall (see Figure 3). ${ }^{28,33}$ This dimple is similar to the type of dimple that forms in a compression-loaded shell at the onset of buckling as observed in tests and is thus a physically meaningful initial imperfection or perturbation. In this analysis-based lower-bound approach, the perturbation load is applied before the axial load is applied and is held constant during the application of the axial load. It has been determined that there exists a range of lateral perturbation loads where the shell buckling load is very sensitive to changes in the magnitude of this perturbation load. However, above a certain threshold value of the perturbation load, the buckling load appears to reach a minimum value or lower bound and is not sensitive to variations in the perturbation load. The buckling load at the threshold value is thought to be a reasonable approximation of a lower bound to the buckling load of the shell. An example of this approach is shown in Figure 4 from reference 28. In particular, Figure 4 a shows the effects of the lateral perturbation load on the global buckling load of the shell and that a lower bound buckling load exists as indicated by the plateau in the curve. The open triangle symbols indicate global buckling and the filled blue triangles are associated with local buckling at the lateral load application point. In addition, the 
local dimple that forms in the shell and the load versus end-shortening response curves (see Fig 4b) are representative of behaviors observed in tests. It should be noted that a stable local dimple can exist in a shell, as suggested by these results, but would be associated with an extreme manufacturing imperfection that likely would not be accepted as flight hardware.

\section{Lateral load, Q}

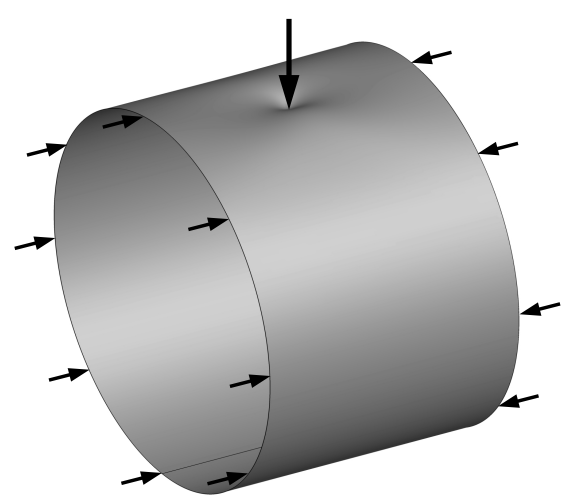

Figure 3. Compression-loaded cylinder with a lateral perturbation load Q.

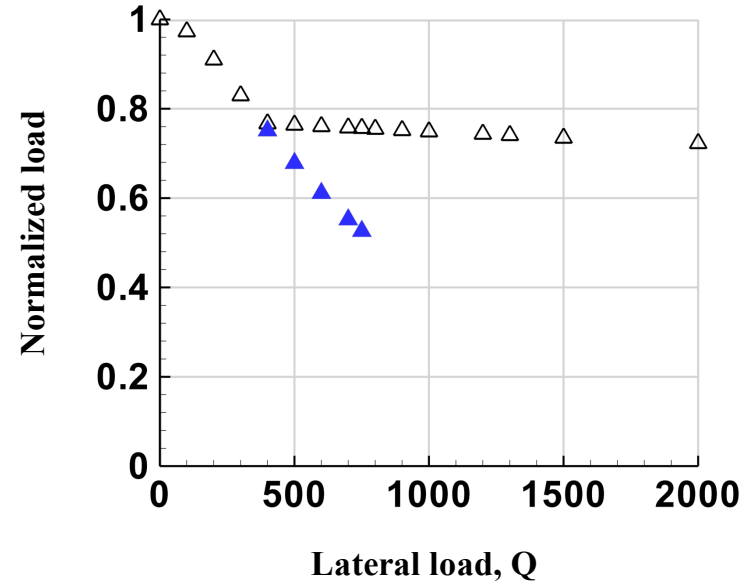

(a)

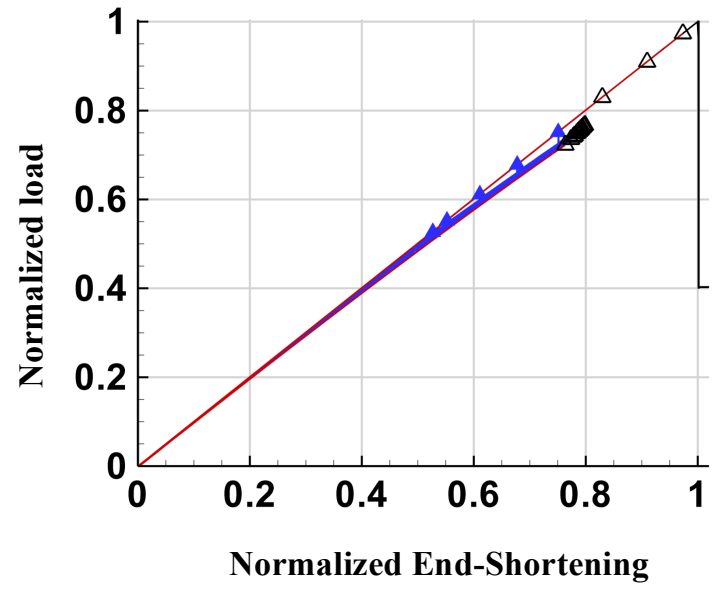

(b)

Figure 4. Predicted buckling loads (a), and load-end-shortening curves (b) for a compression-loaded cylinder for various lateral perturbation load levels.

A similar approach is being used for determining lower-bound buckling loads of shells with nonuniform or imperfect loading conditions that have been observed in testing. Such a boundary imperfection can come about in an as-built structure due to localized manufacturing irregularities or machining tolerances and can cause nonuniform loading in the structure when joined to adjacent structure. Geier et al. ${ }^{33}$ used a shim layer to apply such a boundary imperfection in experimental tests on composite shells. Hühne et al. ${ }^{34}$ also showed that a lower bound of buckling load can be found for this type of imperfection. A loading imperfection can be simulated in the shell by including an axial perturbation load on one of the edges of the shell, as shown in Figure 5. A description of the methods used for predicting lower-bound buckling loads and knockdown factors for geometric imperfections and loading imperfections are given in Ref 
35. In addition, results from these lower-bound buckling knockdown factors are compared to traditional knockdown factors and indicate that these new factors are typically much less conservative.

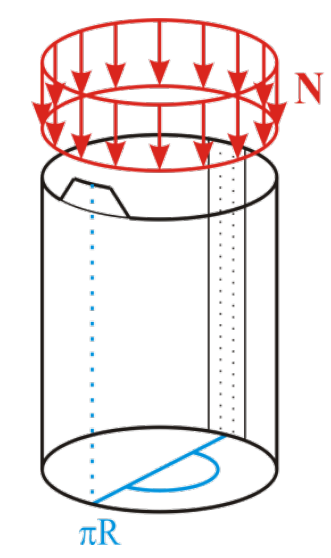

Figure 5. Compression-loaded cylinder with an imperfection on the loaded edge.

\section{Implementation and Expected Benefits}

It is obvious that one main benefit of the updated and less conservative knockdown factors is the potential for significant mass reduction in launch vehicle structures that can translate into increased payload. However, with the current emphasis on affordability, it is also important to identify the other potential benefits that are related to cost and sustainability, and these benefits are described here briefly.

These new factors and recommendations are expected to reduce cost during the design and certification processes in several ways. First, using factors that are tailored (appropriate) for the design of choice will reduce the risk of getting stuck in a poor (less than optimal) design at the beginning that requires redesign. For example, current design factors do not account for several important design features that can affect buckling or imperfection sensitivity including shell length and buckling-critical joint features. In addition, designs with high twisting stiffness, such as isogrid-stiffened designs, may be penalized due to the current methods used to calculate $t_{\text {eff. }}$. The equation for $t_{\text {eff }}$ in SP-8007 is given by

$$
t_{\text {eff }}=\sqrt[4]{\frac{D_{11} D_{22}}{A_{11} A_{22}}}
$$

and is used in the calculation of the knockdown factor presented in Section II. This particular representation of $t_{\text {eff }}$ does not account for the twisting stiffness $D_{66}$, which is typically very large in isogrid-stiffened structures and provides a large contribution to its resistance to buckling. Second, the updated knockdown factors will include manufacturing tolerance variables for the geometric imperfection and loading imperfection, and will enable performance versus manufacturing cost trades. For example, the effects of changing build tolerances such as machining and fit-up tolerances that can contribute to non-uniform loading, or weld distortion 
tolerances which affect the shell geometry can be assessed quickly in preliminary design by using knockdown factors without resorting to high-fidelity computer simulations. In addition, these knockdown factors will provide for quantified robustness measures. Third, as mentioned previously, the new factors will itemize the effects of several common LV design issues and features, and this will enable a reduction in mass growth during the design process. This can be achieved by allowing the KDF values to evolve in a consistent way as the fidelity and knowledge of the design improves, so that the effects of selected imperfections and design details can be adjusted as modeling details and buckling predictions change. In contrast, the traditional approach assumes that the factor stays constant throughout the design cycle, even after additional knowledge and features (weld lands, cutouts, boundary conditions) are added to the computational model, thus these effects are double book-kept and result in mass growth.

Another important consideration in developing these new factors and recommendations is to assess their impact on vehicle sustainability, e.g. operational costs. It is expected that itemized knockdown factors will enable rapid assessments of non-conformance items such as out-oftolerance interface conditions, excessive weld land distortions, or local geometric anomalies such as dimples. This may be achieved by a simple recalculation of buckling margins with an updated factor value that is appropriate for the non-conformance item. Finally, validated high-fidelity analysis methods and recommendations being developed and validated by SBKF will enable accurate predictions of structural development and qualification testing and has the potential to reduce the amount of testing required by using the analysis as a "virtual test".

\section{Concluding Remarks}

A comprehensive project is in place at NASA to develop and validate the next generation of analysis-based shell buckling knockdown factors and design recommendations for modern LV structures. These new knockdown factors will be based on the results from high-fidelity analysis tools that have been rigorously validated through sub-scale and full-scale structural tests. These new factors will account for the effects of geometric imperfections, loading nonuniformities, orthotropy, longitudinal welds, and combined mechanical, thermal and pressure loads. In addition, these factors will be parameterized in such a way that these individual effects can be added and subtracted as required by the design. It is expected that the new factors will enable significant mass reduction in the next generation of NASA LV as well as reduce development and operational costs.

\section{Acknowledgments}

This work was conducted as part of the NASA Engineering and Safety Center (NESC) Shell Buckling Knockdown Factor Project, NESC Assessment \#:07-010-E.

\section{References}

1. von Kármán, T. and Tsien, H. S., "The Buckling of Thin Cylindrical Shells Under Axial Compression," Journal of the Aeronautical Science, Vol. 8, No. 8, June 1941, pp. 303-312.

2. Donnell, L. H. and Wan, C. C., "Effect of Imperfections on Buckling of Thin Cylinders and Columns Under Axial Compression.," Journal of Applied Mechanics, Vol. 17, No. 1, March 1950, pp. 73-83.

3. Koiter, W. T., On the Stability of Elastic Equilibrium. (in Dutch), H. J. Paris, Amsterdam, Holland, 1945; translation available as AFFDL-TR-70-25, February, 1970, Wright-Patterson Air Force Base.

4. "Buckling of Thin-Walled Circular Cylinders," NASA Space Vehicle Design Criteria, NASA SP-8007, September 1965 (Revised 1968). 
5. "Buckling of Thin-Walled Truncated Cones," NASA Space Vehicle Design Criteria, NASA SP-8019, September 1968.

6. "Buckling of Thin-Walled Doubly Curved Shells," NASA Space Vehicle Design Criteria, NASA SP8032, August 1969.

7. Peterson, James P.: Buckling of Stiffened Cylinders in Axial Compression and Bending - A Review of Test Data. NASA TN D-5561. 1969.

8 "Isogrid Design Handbook," NASA CR-124075, February 1973.

9. Hilburger, M.W.; Nemeth, M.P.; Starnes, J.H.,Jr.: Shell Buckling Design Criteria Based on

Manufacturing Imperfection Signatures, AIAA Journal, vol. 44, no. 3, March 2006.

10. Huhne, C.; Rolfes, R.; Breitbach, E.; and Tessmer, J.: Robust Design of Composite Cylindrical Shells

Under Axial Compression - Simulation and Validation, Thin-Walled Structures, vol. 46, no. 7-9, pp. 947962, 2008.

11. Degenhardt, R.; et al.: Investigations on Imperfection Sensitivity and Deduction of Improved

Knockdown Factors for Unstiffened CFRP Cylindrical Shells, Composite Structures, vol. 92, no. 8, pp.1939-1946, 2010.

12. "Space Engineering - Buckling of Structures," European Cooperation for Space Standardization, Handbook ECSS-E-HB-32-24A, ECSS Secratariat, ESA-ESTEC Requirements and Standards Division, March 2010.

13. Dickson, J.N.; Brolliar, R.H.: The General Instability of Ring-Stiffened Corrugated Cylinders Under Axial Compression. NASA TN D-3089, 1966.

14. Meyer, R.R.: Buckling of Ring-Stiffened Corrugated Cylinders Subjected to Uniform Axial Compression Load and Bending. Rept. DAC-60698, Douglas Aircraft Co., 1967.

15. Peterson, J.P.; Dow, M.B.: Compression Tests on Circular Cylinders Stiffened Longitudinally by Closely Spaced Z Section Stringer. NASA Memo 2-12-59L, 1959.

16. Peterson, J.P.; Whitley, R.O.; Deaton, J.W.: Structural Behavior and Compressive Strength of Circular Cylinders with Longitudinal Stiffening. NASA TN D-1251, 1962.

17. Becker, H.; Gerard, G.; and Winter, R.: Experiments on Axial Compressive General Instability of Monolithic Ring-Stiffened Cylinders. AIAA J., vol 1, no 7, July 1963, pp. 1614-1618.

18. Card, M.F.; Jones, R.M.: Experimental and Theoretical Results for Buckling of Eccentrically Stiffened Cylinders. NASA TN D-3639, 1966.

19. Milligan, R.; Gerard, G.; and Lakshinikanthan, C.: General Instability of Orthotropically Stiffened Cylinders Under Axial Compression. AII J., vol 4, no. 11, 1966, pp. 1906-1913.

20. Singer, J.: The Influence of Stiffener Geometry and Spacing on the Buckling of Axially Compressed Cylindrical and Conical Shells. Preliminary Preprint Paper, $2^{\text {nd }}$ IUTAM Symposium on the Theory of Thin Shells, Copenhagen, 1967.

21. Meyer, R.R.: Buckling of $45^{\circ}$ Eccentric-Stiffened Waffle Cylinders. J. Roy. Aeronaut. Soc. (Tech Notes) vol. 71, no. 679, 1967, pp. 516-520.

22. Peterson, J.P.; and Anderson, J.K.: Bending Tests of Large-Diameter Ring-Stiffened Corrugated Cylinders. NASA TN D-3336, 1966.

23. Anderson, J.K.: Bending Tests of Two Large-Diameter Corrugated Cylinders with Eccentric Ring Stiffeners, NASA TN D-3702, 1966.

24. Card, M.F.: Bending Test of Large-Diameter Stiffened Cylinders Susceptible to General Instability, NASA TN D-2200, 1964.

25. Lovejoy, Andrew E., Chunchu, Prasad B., and Hilburger, Mark W.. "Ares-V Design Study: The Effects of Buckling Knockdown Factors, Internal Pressure, and Materials." NASA/TM-2011-217061, February, 2011.

26. Lovejoy, Andrew E., Chunchu, Prasad B., and Hilburger, Mark W.. "Ares-I Upper Stage Design Study: The Effects of Buckling Knockdown Factors and Internal Pressure." NASA/TM-2010-216848, October, 2010.

27. Lovejoy, Andrew E., Hilburger, Mark W., and Chunchu, Prasad B. " Effects of Buckling-Knockdown Factor, Internal Pressure and Material on the Design of Stiffened Cylinders." AIAA-2010-2778, 51st 
AIAA/ASME/ASCE/AHS/ASC Structures, Structural Dynamics, and Materials Conference, Orlando, Florida, Apr. 12-15, 2010.

28. Haynie, Waddy T., and Hilburger, Mark W., "Comparison of Methods to Predict Lower Bound Buckling Loads of Cylinders Under Axial Compression," AIAA Paper 2010-2532, $51^{\text {st }}$

AIAA/ASME/ASCE/AHS/ASC Structures, Structural Dynamics, and Materials Conference, 12 - 15 April 2010, Orlando, Florida.

29. Haynie to be presented at the $53^{\text {rd }}$ AIAA/ASME/ASCE/AHS/ASC Structures, Structural Dynamics, and Materials Conference, Honolulu, HI, Apr. 23-26, 2012.

30. Hilburger, M.W.: Shell Buckling Knockdown Factor $-1^{\text {st }}$ Annual Workshop 14-15 October, 2009, NASA/TM-2010-216850, October, 2010. (Distribution limited)

31. Hilburger, M.W.; Starnes, J.H., Jr.: Effects of Imperfections on the Buckling Response of

Compression-Loaded Composite Shells, International Journal of Non-Linear Mechanics, vol. 37, no. 4-5, pp. 623-643, 2002.

32. Hilburger, M.W.: Static Buckling Tests of Aerospace Vehicle Structures, NASA/TM-2010-216849, October, 2010.

33. Geier, B.; Klein, H.; and Zimmermann, R.: Experiments on the Buckling of CFRP Cylindrical Shells Under Non-Uniform Axial Load, presented at the International Conference on Composite Engineering, New Orleans, LA, 1994, pp. 28-31.

34. Huhne, C.; Zimmermann, R.; Rolfes, R.; and Geier, B.: Sensitivities to Geometrical and Loading Imperfections on Buckling of Composite Cylinrical Shells, presented at the European Conference on Spacecraft Structures, Materials and Mechancial Testing, Toulouse, France, 2002.

35. Kriegesmann, B.; Hilburger, M.W.; and Rolfes, R.: The Effects of Geometric and Loading Imperfections on the Response and Lower-Bound Buckling Load of a Compression-Loaded Cylindrical Shell, to be presented at the $53^{\text {rd }}$ AIAA/ASME/ASCE/AHS/ASC Structures, Structural Dynamics, and Materials Conference, Honolulu, HI, Apr. 23-26, 2012. 